\title{
Un modelo y un método para la transformación de la biblioteca escolar en centro de recursos de enseñanza y aprendizaje
}

\author{
Transforming the school library into a teaching \\ and learning resource center: \\ a model and a method
}

\author{
Miguel Ángel MARZAL' \\ María José DÍAZ² \\ Javier CALZADA ${ }^{1}$
}

\section{Resumen}

El propósito de este trabajo es presentar un modelo y un método para la transformación de la biblioteca escolar en Centro de Recursos para la Enseñanza y el Aprendizaje que permita avanzar en la cultura bibliotecaria y la integración curricular de la alfabetización informacional en los centros públicos de enseñanza secundaria de la Comunidad de Madrid. Dichos modelo y método se proponen a partir de un estudio de la realidad y expectativas de la comunidad educativa madrileña, para el cual se realizaron encuestas a directores, profesores, responsables de bibliotecas y alumnos de 10 Institutos de Educación Secundaria. Los resultados del estudio sugieren la necesidad de un modelo de Centro de Recursos para la Enseñanza y el Aprendizaje integrado en la actividad escolar a cargo de un bibliotecario escolar cualificado y a tiempo completo, capaz de colaborar con los docentes en el desarrollo del currículo así como con otras bibliotecas. Para su implementación se propone un método de evaluación basado en indicadores relativos a aspectos clave de su actuación, así como una serie de aplicaciones prácticas del Centro de Recursos para la Enseñanza y el Aprendizaje en el ámbito escolar. Se concluye, finalmente, que el Centro de Recursos para la Enseñanza y el Aprendizaje así definido puede contribuir a potenciar la inclusión digital de los escolares.

Palabras-clave: Alfabetización en información. Biblioteca escolar. Centro de recursos para la enseñanza y el aprendizaje. Indicadores educativos

\begin{abstract}
The purpose of this article is to present a model and a method with the aim of transforming the school library into a Teaching and Learning Resource Center that will allow advancement in the curricular integration of information literacy within Secondary Education Public Centers of the Comunidad de Madrid, Spain.The said model and method are proposed as a result of a study of the reality and expectations of the local educational community, based on a survey of headmasters, teachers, librarians and students from 10 high schools. The results of the study suggest the need for a Secondary Education Public Centers model that is integrated into the school activity, with a full-time qualified school

1 Profesores Doctores, Universidad Carlos III de Madrid, Departamento de Biblioteconomía y Documentación. Campus de Getafe, Calle Madrid, 126, 28903, Getafe, Madrid, España. Correspondencia a nombre de/Correspondence to: M.A. MARZAL. E-mail: <mmarzal@bib.uc3m.es>.

2 Profesora, Instituto de Enseñanza Secundaria El Escorial. Madrid, España.

Recebido el dia 27/4/2012, re-apresentado en 25/8/2012 e aceptado para su publicación el 8/8/2012.
\end{abstract}


librarian in charge of it, and able to collaborate with teachers in curriculum development, as well as with other libraries. To implement this model, we propose an evaluation method based on indicators related to key aspects of the Secondary Education Public Centers performance as well as several practical applications in schools. Finally, we conclude that a Secondary Education Public Centers, thus defined, may contribute to foster digital inclusion among students.

Keywords: Information literacy. School library. Teaching and learning resources centers. Educational indicators.

\section{Introducción}

El notorio progreso de las Tecnologías de la Información y la Comunicación (TIC) y la Web ha ido impulsando a las bibliotecas hacia una redefinición imprescindible de modelo, funciones y proyección sobre su comunidad de usuarios. Los tradicionales elementos de definición de una biblioteca - también en aquellas con una dimensión educativa - se han transformado: la colección incorpora materiales didácticos que se enriquecen con los recursos y los sitios educativos Web, pero también los objetos digitales educativos y los objetos de aprendizaje, cuyos caracteres y funciones propias de la Web Semántica se orientan a competencias y exige en la biblioteca un tratamiento propio; la organización de una colección digital, en la que las nociones de autoría (edición digital colaborativa, permanente, interactiva), materia (diluida por la asociatividad y transversalidad del hipertexto) e identificación (el contenido Web posee rasgos muy distintos del texto) son muy diferentes y reclaman una gestión de contenidos; los servicios deben orientarse hacia las redes sociales, pero también hacia una Educación que fomente no sólo la producción de conocimiento, sino también su generación mediante la formación por competencias en el aprendizaje permanente y el aprendizaje colaborativo mediante la alfabetización en información; la primacía al usuario individual, atendiendo a sus necesidades, actitudes o consumo de información, de modo que en el ciberespacio la biblioteca debe convertirse en la Unidad de Información de cada uno.

Este contexto ha cimentado un movimiento que considera necesaria la transformación de la biblioteca en centro de recursos. ¿Qué significa? No se trataba de un simple cambio de nombre ni la desaparición de la biblioteca en su definición tradicional, de modo que emergió la noción de biblioteca híbrida, para indicar la doble dimensión tradicional y digital. El desarrollo de las potencialidades del mundo digital y virtual, en todo caso, permitió que entre los años 2003 y 2005 hubiese un serio esfuerzo de definición y conceptualización, que ha dado origen a investigaciones y literatura científica en torno a la biblioteca digital y la biblioteca virtual. Se sentaban las bases para un modelo, medios y aplicación en entornos de información digital educativa. De este modo se acuña el término y concepto de biblioteca digital educativa en el ámbito norteamericano el que, cuando conecta con el nuevo modelo educativo competencial para la sociedad del conocimiento, inicia su conversión en Learning Resources Centres (LCR) para el ámbito anglosajón, o Centros de Recursos para el Aprendizaje e Investigación (CRAI) en universidades, Centro de Recursos para la Enseñanza y el Aprendizaje (CREA) en educación preuniversitaria (por cambiar investigación por enseñanza) en el ámbito continental europeo. La biblioteca no desaparece, sino que se convierte en un recurso más.

La transformación es más necesaria, pues la Educación ha debido evolucionar hacia un modelo competencial en el que los "centros de recursos" son un elemento clave en el proceso. Se hace preciso, pues, determinar qué debemos entender por CREA, en tanto que centro de recursos que integra la biblioteca escolar, dentro de un modelo educativo competencial preuniversitario: "debe ser un nuevo espacio educativo dinámico, no mero gestor de recursos educativos sino ámbito para una metodología didáctica activa, interdisciplinar y adaptada a la diversidad de entornos y aprendizajes; centro suministrador, organizador de saberes y potenciador del autoaprendizaje, no complemento del currículum académico, sino parte integrante de él" (Marzal; Cuevas, 2007, p.60). El CREA posee como funciones específicas: servir de soporte a la enseñanza, desarrollando la autonomía personal en los propios procesos de aprendizaje a través de la Alfabetización en Información; desarrollar el blended learning y fomentar las comunidades virtuales de aprendizaje; facilitar el acceso a recursos digitales educativos mediante una eficaz organización y gestión de contenidos; ser centro 
productor de contenidos educativos, en todo formato; ser espacio de socialización.

\section{Un proyecto para un modelo de transformación: hipótesis y objetivos}

Si bien el concepto, organización y funciones de la biblioteca escolar están determinados en lo estructural, lo cierto es que, sin reconocimiento específico, el modelo de biblioteca escolar es difuso y disfuncional, por lo que su conversión modélica a CREA parece también etéreo.

La definición de un posible procedimiento metódico de transformación parece convenir que se inspire en el que se ha ensayado para las bibliotecas universitarias hacia el CRAI en España, sin una simple extrapolación. En este sentido, parece especialmente útil el procedimiento estipulado en el proyecto Biblioteca Universitaria y Centros de Recursos para el Aprendizaje y la Investigación (BUCRAI), liderado por M. Area, sufragado por el Ministerio de Educación de España y con apoyo de la Conferencia de Rectores de Universidades Españolas (CRUE) y la Red de Bibliotecas Universitarias (REBIUN) (Area et al., 2005). En él se definía el CRAl, sus funciones, elementos y potencialidades, y se definían también algunos instrumentos para un estudio de campo ante el inicio del proceso de transformación y la actitud de los agentes críticos implicados, así como las expectativas de la comunidad universitaria. El proceso de transformación comienza con un proceso de convergencia de servicios bibliotecarios, informáticos, audiovisuales. Es una etapa "infraestructural" donde se crea un único centro que aglutina todos los servicios $\mathrm{TIC}$, se dispensa un servicio integrado a la comunidad educativa y se genera una plataforma integrada de información digital. En una segunda fase se produce un proceso de confluencia de servicios hacia la gestión de contenidos, las redes sociales y biblioteca 2.0, la formación en alfabetización en información.

En este contexto y bajo estos parámetros se planteó el Proyecto de Investigación, fundamento para la Licencia por Estudios concedida por la Consejería de Educación de la Comunidad de Madrid (Madrid, 2007, online), "Propuestas dirigidas a los Centros Públicos de Secundaria de la Comunidad de Madrid para la incorporación de un Modelo de transformación de
Biblioteca Escolar tradicional en Centro de Recursos para la Enseñanza y el Aprendizaje". La hipótesis de trabajo se fundamentaba en que la transformación de biblioteca escolar en CREA proporcionará al alumno los instrumentos y las herramientas necesarios para la adquisición de las competencias básicas, según recoge la ORDEN ECI/2220/ 2007, de 12 de julio, por la que se establece el currículo y se regula la ordenación de la Educación Secundaria Obligatoria, que en su Anexo I desarrolla las competencias básicas que el alumnado deberá haber adquirido en el marco de la propuesta realizada por la Unión Europea (España, 2007).

El objetivo general del proyecto se asienta en proponer a los Institutos de Educación Secundaria herramientas y estrategias que permitan a la comunidad educativa, a través de las bibliotecas escolares, desarrollar las acciones educativas en materia de alfabetización en información y las competencias adecuadas para comprender la necesidad de información y de ese modo avanzar en la cultura de uso de la biblioteca escolar, siendo para ello necesaria su transformación en CREA. El objetivo general se proyecta en objetivos específicos: definir un modelo conceptual de transformación de biblioteca escolar en CREA que sea adaptable a cualquier centro público de Educación Secundaria; diseñar los cuestionarios pertinentes para plantear el mejor modelo de transformación, asociados a indicadores adecuados que permitan la correcta evaluación de las actuaciones llevadas a cabo en las fases de implantación del modelo; proponer programas formativos de alfabetización en información para integrar de forma eficaz los recursos educativos Web en el currículo, así como actividades para favorecer en los alumnos la adquisición de habilidades para el desarrollo de la alfabetización digital; proponer herramientas para organizar los contenidos de los recursos educativos y elaborar estrategias que conduzcan al aprendizaje autónomo de los alumnos; contribuir a que la biblioteca - CREA - sea la herramienta que proporcione la igualdad de oportunidades para todos los alumnos contra la brecha digital y el fracaso escolar.

\section{Planificación, materiales, métodos e instrumentos del proyecto}

La planificación del proyecto se desarrolló en tres etapas: 
En la Etapa / se llevó a cabo la búsqueda y recopilación de fuentes de información más relevantes para determinar el "estado del arte".

En la Etapa // se analizó la situación de las bibliotecas escolares en tres fases: en la primera se recogió información sobre las bibliotecas escolares de la Comunidad de Madrid dentro de la Consejería de Cultura y Deportes, se contactó con los centros bilingües de la Comunidad para determinar quienes colaborarían en el proyecto, y se elaboraron los cuestionarios para los agentes que intervienen en la biblioteca (equipo directivo, responsable de la biblioteca, profesores y alumnos) para realizar un análisis y un diagnóstico de la situación de partida de la biblioteca de cada centro; en la segunda fase se realizó la entrevista con directores y responsables para exponerles los objetivos del proyecto y entregarles la información impresa y los cuestionarios, junto con 4 ó 5 visitas a cada centro, durante el proyecto, para llevar a cabo la observación sistemática y crítica del funcionamiento de la biblioteca con un esquema modélico de actuaciones, procediéndose finalmente a recoger los cuestionarios ya cumplimentados (469 alumnos, 10 directores, 10 responsables de biblioteca y 39 profesores). La tercera fase consistió en la extracción de datos de los cuestionarios (se enviaron los resultados de los cuestionarios de los alumnos a los centros, con el fin de que los responsables conozcan la opinión que tiene el alumnado de su biblioteca con respecto a los servicios y recursos de los que disponen y permitirles así detectar cuáles son sus debilidades y fortalezas) y luego en análisis estadístico de los datos y su resultados, con categorización y valoración de los mismos.

En la Etapa III se acometió la propuesta de un modelo de transformación de biblioteca tradicional en CREA basado en alfabetización en información, junto con una propuesta de indicadores para evaluar el modelo de transformación, así como propuestas para la formación del profesorado y de programas de actividades entre los alumnos respecto a la alfabetización en información y digital.

Para el correcto desarrollo de esta planificación se han aplicado diversas herramientas metodológicas, cada una con sus propias fuentes y materiales de trabajo: descriptiva, por la recopilación de los recursos necesarios para la elaboración del trabajo mediante consulta de la literatura científica suficiente para el planteamiento de un marco conceptual, así como para un estudio de campo mediante entrevistas; aplicada, por la elaboración de cuestionarios estructurados e híbridos con preguntas cerradas y abiertas de naturaleza cuantitativa y cualitativa, cuyo análisis ha permitido conocer la situación de la biblioteca de cada centro estudiado y así definir las pautas de transformación más adecuada, datos complementados por la observación sistemática y crítica del funcionamiento de cada biblioteca desde un esquema modélico de actuaciones; deductiva, por el análisis de los resultados, asegurando la relevancia y utilidad de los mismos para finalmente presentar las conclusiones destinadas a favorecer un diagnóstico de debilidades y fortalezas con respecto a los servicios y recursos de los que disponen para poder planificar las actuaciones más oportunas a realizar, así como acciones referidas a la alfabetización en información, proponiendo un posible sistema de evaluación del proceso.

La viabilidad y solidez científica en los resultados del proyecto exigió la definición de una muestra. El elevado número de Institutos de Educación Secundaria de la Comunidad de Madrid (318) aconsejó determinar los criterios de selección en la muestra estadística de Centros: todas las Direcciones de Área Territorial (DAT) de la Comunidad deberían estar representadas; todos debían ser Centros de bilingüismo, esto es, que tuviesen autorizada una Sección Lingüística de Lengua Inglesa; para hacer más eficaz el proyecto acotamos el universo estadístico en los Centros que ya tuvieran biblioteca central, apta para acoger un modelo de transformación a CREA. Es preciso reseñar, como variables, la gran diversidad en el total de alumnos y en la distribución de los mismos por niveles: si bien todos los Centros imparten Enseñanza Secundaria y Bachillerato, seis ofrecen Formación Profesional y uno Enseñanza a Distancia - o Centro para la Innovación y Desarrollo de la Educación a Distancia (CIDEAD). El número total de alumnos matriculados en los Centros seleccionados ha sido de 9697, con una media de 969 por Centro.

Como instrumento básico para el desarrollo del proyecto se utilizaron los cuestionarios. Para ello se elaboraron cuatro cuestionarios distintos según el agente-usuario al que iba dirigido: director, responsable de biblioteca, profesorado y alumnos, por percibir la 
biblioteca desde puntos de vista muy diferentes. Se solicitó a los centros que los cuestionarios fueran cumplimentados por cinco profesores que representasen a Departamentos diferentes y por 15-20 alumnos de cada uno de los niveles de enseñanza. De los 50 cuestionarios posibles del profesorado han sido respondidos 39 y de los 500 posibles de los alumnos han sido respondidos 469, repartidos de la siguiente manera: 143 de $1^{\circ}$ Ciclo, 171 de $2^{\circ}$ Ciclo y 145 de Bachillerato. Los cuestionarios constan mayoritariamente de preguntas de carácter cerrado, aunque también se ha dado la opción de poder elegir más de una respuesta en varias preguntas, especificar otras posibilidades o responder a las causas que motivan dichas respuestas, convirtiéndose esta última opción en preguntas mixtas (carácter cerrado-abierto). En los cuestionarios se buscó contemplar las dimensiones de la biblioteca escolar más pertinentes para el proyecto: modelo de biblioteca existente, uso CREA de la biblioteca, gestión de Recursos Humanos, acceso a los recursos (equipamiento, funcionamiento, disponibilidad [...]), grado de satisfacción de los usuarios. Finalmente se planteó una misma pregunta de carácter abierto: ¿Cuál sería su modelo ideal de biblioteca?

Los datos procedentes de los cuestionarios se recogieron en unas hojas de cálculo del programa MS Excel, reflejando todos los datos cuantitativos en frecuencias y porcentajes para el posterior análisis de los resultados y la inclusión de gráficos. Se ha estudiado cada pregunta de forma aislada para después establecer relaciones entre las preguntas referentes a un mismo contenido y entre las preguntas que, siendo iguales, ofrecen distinta respuesta según el usuario que responda. Luego se ha procedido a la categorización de los resultados de las preguntas de carácter cerrado dentro del contexto del parámetro al que hace referencia y desde la perspectiva de todos los agentes-usuarios cuando se ha requerido, mientras que para las preguntas de carácter abierto (datos cualitativos) el análisis se ha realizado de forma manual.

\section{Análisis de resultados}

Según se ha indicado, y para su más eficaz tratamiento y análisis, los resultados de las preguntas han sido categorizados bajo los siguientes parámetros:

\section{Modelo de biblioteca}

Bajo este parámetro se ha analizado el tipo y proyecto de biblioteca, la evaluación del proyecto, los servicios de la biblioteca, las actividades del centro en la biblioteca, las actividades propias de la biblioteca, la adecuación de los fondos, la colaboración con otras bibliotecas (de otros centros, públicas etc.), la participación en Redes o Asociaciones y la participación en Congresos.

Respecto al tipo de biblioteca, la situación parece saludable. Todos los centros disponen de una biblioteca escolar central, pero además un 50\% la complementan con bibliotecas de Departamento, incluso un 30\% también cuenta con biblioteca de aula. Aún más relevante es la existencia de un proyecto de biblioteca - en un 90\% de los casos-, e incluso parece mayoritaria (si bien en una proporción escasamente suficiente, 60\%) la existencia de evaluaciones del proyecto de biblioteca. Para una biblioteca escolar es de la mayor importancia la inclusión del proyecto de biblioteca en los documentos oficiales del centro, detectándose un fenómeno significativo: el proyecto está 100\% incluido en la Programación General Anual (PGA), respondiendo a la normativa general; sin embargo, al acudir a los documentos didácticos, el proyecto recoge un 70\% de los Proyectos Educativos de Centro (PEC) y un 30\% de los Proyectos Curriculares (PC). El uso y funcionamiento de la biblioteca están también incluidos en el Reglamento de Régimen Interior (RRI) en el $90 \%$ de los centros; un 10\% la recoge, además, en la Agenda Escolar.

Respecto a los servicios que ofrece la biblioteca, se presentó un repertorio de aquellos masivamente presentes en las bibliotecas escolares. Excepto el servicio de reprografía, del cual ninguna biblioteca dispone, el resto se desglosa de la siguiente manera: el 100\% ofrece los servicios de consulta en sala, acceso a Internet y préstamo a domicilio; un 50\% dispone de hemeroteca e información bibliográfica y de referencia; un 70\% de acceso multimedia; un 60\% de préstamos colectivos; un $30 \%$ presta los servicios de reserva de documentos; noticias y novedades un 20\% y, por último un 10\% ofrece boletín informativo.

La función que el centro decida dar a su biblioteca es un indicador principal sobre el modelo de biblioteca escolar, por ello es fundamental conocer las actividades 
realizadas en la biblioteca. Desde luego es masivo el uso del espacio bibliotecario, por parte del centro, como multifunción: el 100\% de los Institutos de Enseñanza Secundaria (IES) utilizan la biblioteca para realizar diversas actividades del centro, según el calendario escolar de compromisos. En todo caso, se reconoce el uso del espacio bibliotecario para actividades culturales (el $30 \%$ ), fomento del uso de la biblioteca (el 20\%), actividades complementarias (ACES), prácticas de alumnos, tutorías, retrasos y sanciones, intercambios, recitales de poesía y redacción de la revista del IES.

Sin embargo, la percepción del modelo a través de actividades se perfila mejor cuando se abordan las actividades que los distintos agentes del centro escolar creen que debe acoger la biblioteca escolar: Ios responsables de la bibliotecay los directores. La percepción sigue un comportamiento interesante: para los directores la actividad más importante es la promoción lectora, pero para los responsables de biblioteca es la conservación de fondos; sin embargo, y en proporción más similar, ambos agentes consideran que la segunda actividad más relevante es la formación de usuarios, algo realmente importante. De hecho, el 30\% de los Centros reconoce reservar un tiempo de los alumnos para actividades de formación de usuarios y lectura en la biblioteca.

También es significativo en la definición de modelo, la adecuación de los fondos de la biblioteca a las necesidades personales, sociales y de ocio de la comunidad educativa, así como la relevancia de los materiales teniendo en cuenta las características actuales de cada centro. En este caso, prácticamente un $77 \%$ del profesorado admite que responde bien a sus necesidades docentes.

Finalmente, el modelo de biblioteca que se perfila debe definirse respecto a la cooperación con otras bibliotecas, asociaciones o Redes. Los resultados, en este caso, son poco alentadores: un 10\% colabora con las bibliotecas de otros centros educativos y mantiene contacto con Asociaciones de Bibliotecas Escolares, mientras que otro 10\% colabora con la Biblioteca Pública participando, además, en la Red de Bibliotecas Públicas de la Comunidad de Madrid por ser Centro Prioritario. Mucho más activa es la extensión bibliotecaria, ya que la participación en concursos, eventos, exposiciones o actividades referentes al libro y a las bibliotecas atrae a un $80 \%$.

\section{Percepción del CREA}

Se trata de analizar si la comunidad educativa tiene conciencia de lo que es, puede suponer y caracteriza al CREA, como una evolución de la biblioteca escolar. Atendiendo al concepto, es llamativo el acuerdo muy amplio entre toda la comunidad educativa en considerar su biblioteca como un espacio educativo de documentación (100,0\% de los responsables y de los directores, y un $94,9 \%$ del profesorado) y como espacio de información y formación (90,0\% de los directores, 80,0\% de los responsables, $94,9 \%$ del profesorado). Aún más, es firme la convicción de que uno de los objetivos de la biblioteca es apoyar el proceso de enseñanza-aprendizaje (87,2\% del profesorado y el $100,0 \%$ de los responsables y directores). Esta percepción de la biblioteca escolar como una herramienta eficaz para facilitar su proceso de enseñanza-aprendizaje, aún mayoritaria, no es tan firme entre los alumnos (entre un 70 y $75,0 \%$ ), dependiendo del nivel educativo de estos: más alto entre los pequeños y más reducido entre los mayores, alumnos de Bachillerato.

Partir de este concepto de la biblioteca escolar como instrumento educativo muy relevante pareció la mejor base para fundamentar su transformación en CREA. Incluso como preámbulo se formuló la pregunta sobre si la biblioteca escolar ejerce en algún servicio como CREA. Un 40\% de los directores, aunque de éstos sólo un 20\% de los responsables, respondían afirmativamente. Bajo esta premisa, parecía adecuada una batería de preguntas sobre los planes para avanzar en la organización de un CREA, lo que permitía, además, constatar si existía una concepción adecuada en torno a lo que es y supone un CREA.

Las respuestas demostraron la brecha entre el diseño y la realidad. Si un 70\% de los directores afirmaban que el proceso transformador se había iniciado entre 2007 y 2009 por estímulo de planes estatales y autonómicos, la opinión sólo era seguida por un 50\% de los responsables. Parecía lógica una pregunta de confirmación referida a en qué había consistido esa transformación. Las respuestas fueron muy variadas, demostración de que no existe un procedimiento ni método normalizado de transformación, porque se reconocieron varios estímulos transformadores (informatización, creación de un equipo estable, incorporación del proyecto de biblioteca al 
Projecto Educativo de Centro (PEC), al Projecto Curricular de Centro (PCC) y a los proyectos educativos del centro; formar parte de la Red de Bibliotecas Públicas de la Comunidad de Madrid).

La envergadura de la transformación, la inexistencia de un método de cambio, parecía necesitar una pregunta sobre la gestión de expectativas, específicamente qué se espera de la transformación. Un $50,0 \%$ de los directores y un 60,0\% de los responsables reconocían que el CREA debe convertir la biblioteca en un instrumento de apoyo a los proyectos curriculares, de lectura y de investigación del centro, debe funcionar como un banco de recursos documentales de todo tipo, debe ser un instrumento de mejora y fomento de información educativa por centralizar sus recursos e incrementar los usuarios, así como debe ser una oportunidad de visibilidad fuera del centro. La respuesta más significativa, sin embargo, es la que podían proporcionar los docentes y que parece alentadora: un 69,2\% admite que el CREA favorecerá la adquisición de competencias básicas entre sus alumnos, pero, aún más, un 70,5\% considera que el CREA debe ser un espacio educativo, dinámico y social.

Una vez constatada la percepción del modelo de biblioteca escolar y la "imagen" del CREA, entre la comunidad educativa, el cuestionario se desplazó a recabar datos sobre el uso de los recursos informativos o la formación en alfabetización en información. Los grupos-meta críticos, en esta fase, sin duda, eran profesores y alumnos, a quienes se les consultó sobre el uso de los fondos de la biblioteca:

Sólo un 46,2\% del profesorado utiliza los recursos "Gutenberg" (tradicionales) en la biblioteca cuando acuden con sus alumnos (un 53,8\% no, pero además un 74,4\% no utiliza los recursos Web). Uno de los pilares de uso eficaz educativo del CREA parece quedar eliminado. El alumnado es más activo en el uso de fondos. Entre un 70 y $72,0 \%$, según niveles educativos, utilizan los fondos clásicos, y entre un 54,5\% y 62,6\% los recursos Web, datos algo frustrantes. Mucho más esperanzador es constatar el objetivo didáctico del profesorado al usar una biblioteca hacia el CREA: un 82,1\% afirma enseñar habilidades de uso de la información y trabajo documental basado en la utilización de diversas y múltiples fuentes. También se hacía necesario preguntar sobre la formación en el método científico mediante la elaboración de "trabajos académicos", aunque aquí la divergencia es muy significativa: entre un $42,0 \%$ de los pequeños y un 56 a $48,0 \%$ de alumnos medianos y mayores utilizan los recursos disponibles en la biblioteca, mientras que sólo lo hace un 28,2\% del profesorado.

El profesorado, pues, en la práctica, no tiene interiorizada la biblioteca-CREA como instrumento de educación competencial científica, lo que confirma que sólo un 53,8\% usa mapas conceptuales como recurso de aprendizaje y un 28,2\% no reconoce el uso didáctico de la biblioteca. Abundando en la idea de que las TIC parecen no estar asociadas al CREA, es curioso que el 87,2\% del profesorado y entre el $67,1 \%$ de los pequeños y el $85,5 \%$ de los alumnos mayores consulta regularmente Internet para documentar y complementar su aprendizaje y labor docente. Los alumnos, incluso, entre el 89 y 91,0\%, según el nivel educativo, decían saber buscar las fuentes de información y seleccionar la información importante, si bien sólo entre el 55 y 65,0\% incluye las fuentes de información en sus trabajos.

\section{Gestión de recursos humanos}

Parámetro referido al personal responsable de la biblioteca-CREA. Los resultados son rotundos: un 50\% tiene un responsable fijo y estable, mientras que un 30\% cuenta con un responsable que cambia cada curso según las necesidades de horario, disponiendo también de la aportación de profesores con horas complementarias. El porcentaje de Centros que disponen de varios profesores con horas complementarias de biblioteca en su horario es del $60 \%$.

Los agentes críticos, en esta fase del cuestionario, son, sin duda, directores y responsables, cuya percepción de la acción del personal en la biblioteca-CREA es, naturalmente, muy distinto. Para el 60,0\% de los directores existe un equipo de profesores que trabaja de forma coordinada según un proyecto, porcentaje que disminuye hasta un 30,0\% entre los responsables. Respecto a la formación específica en Documentación (grado o máster), un 50,0\% de los responsables la tiene; pero, además, un $74,4 \%$ del profesorado opina que deben tener una formación específica en Documentación, lo que no obsta para que sólo el 33,3\% del profesorado opine que el responsable de la biblioteca tuviera dedicación exclusiva. 
En realidad, o varios responsables suelen constituir un equipo de profesores que trabaja de forma coordinada según un proyecto o bien varios profesores tienen horas complementarias de biblioteca (normalmente guardias).

Durante las horas de dedicación a la biblioteca se llevan a cabo diversas tareas relacionadas con los procesos técnicos, la dinamización, la formación de usuarios etc., constatando que en algunas de las tareas planteadas en el cuestionario como el préstamo, la selección y adquisición de fondos y la catalogación coincide el 100\% de los centros.

Existe, sin embargo, un dato de mayor interés: un 50\% de las bibliotecas abre en horario extraescolar, teniendo como responsables a un monitor del Ayuntamiento que trabaja dentro del Plan de Extensión Educativa o a un profesor del centro, si bien las tareas son las mismas, salvo una atención especial a la ayuda al estudio.

\section{Uso y servicios: grado de satisfacción de los usuarios}

Parámetro dirigido a analizar el horario de la biblioteca, la asistencia de usuarios, su utilización, la distribución de las zonas y la señalización, el equipamiento, los presupuestos, el fondo, el acceso al mismo, el tipo de recursos y la difusión de la información, como también la proyección tangible de la biblioteca sobre la comunidad escolar, por la resolución de necesidades informativas, manifestada en el grado de satisfacción.

En una gran mayoría - 80,0\% -, el horario de la biblioteca comienza con la primera hora de la jornada lectiva, pero quizás es más relevante el horario de cierre, en el que hay una mayor diversidad, indicativo de la vitalidad de la biblioteca: el 40,0\% cierra al final del horario lectivo de la mañana (un 10,0\% más vuelve a abrir en el horario lectivo de la tarde hasta las 19:30), mientras que existe un 30,0\% que permanece abierta hasta las 19:00, las 21:00 ó las 21:50. Un 10,0\% tiene acceso libre a la biblioteca únicamente en los recreos y, por último, un 10,0\% no especifica el horario. La opinión de los usuarios más interesados, profesores y alumnos, es divergente: entre el 60 y 74,1\% entre los alumnos lo ve adecuado, opinión satisfactoria que llega al 82,1\% entre los profesores, evidenciando un uso distinto por ambos colectivos. Los alumnos acuden con diferente frecuencia según los niveles; en el Primer Ciclo es mayor el porcentaje de uso una vez a la semana o todos los días, en el Segundo Ciclo el porcentaje es mayor en una vez al mes, sólo en época de exámenes y sólo cuando el profesor da clase en la biblioteca, y, en el Bachillerato, el porcentaje aumenta considerablemente sólo en época de exámenes. La frecuencia de uso por parte del profesorado acredita que no se visualiza la biblioteca como instrumento educativo en el aspecto práctico:

La organización del espacio de la biblioteca es también un indicador importante de su uso. El cuestionario recogía 11 posibles zonas a distribuir (Préstamo y Gestión; Lectura; Acceso a Internet; Estudio y trabajo; Información general; Audiovisuales; Novedades; Hemeroteca; Referencia; Trabajo en pequeños grupos; Taquillas). Excepto una biblioteca que cuenta con nueve, el resto suele disponer de entre 3 y 6 zonas. Respecto a la señalización de la biblioteca, una mayoría bien representativa, entre el 80 y 90\% tanto de profesores como alumnos, considera que cumple bien su función de orientar con facilidad y ser usada de forma autónoma. Respecto al equipamiento informático, audiovisual y multimedia del que disponen las bibliotecas nos ofrece una realidad muy diversa, si bien todas las bibliotecas tienen conexión Internet, el no de ordenadores varía entre un 20\% que tiene 8-9 ordenadores y otro $20 \%$ un solo ordenador (el $60 \%$ restante tiene entre 2 y 7 ordenadores, la mayoría entre 3 y 4). Un $60 \%$ tiene lector de código de barras, un 50\% cañón de proyección y un 20\% dispone de pantalla portátil y escáner.

El presupuesto, evidentemente, es otro indicador fundamental para detectar el grado de compromiso del centro con este servicio, así como de la funcionalidad que se le otorga. El 90\% tiene incluida una dotación anual para la biblioteca en los presupuestos del centro, un dato esperanzador, pero lo cierto es que, cuando los centros reconocen una partida bien definida para la biblioteca, ésta oscila entre $1500 €$ y los $4000 €$.

El volumen de los fondos en las bibliotecas escolares consideradas varía entre un 20\%, que supera los 15000 registros, y un 10\% con 2 500-5 000, si bien más del 50\% oscila entre los 5000 y 10000 registros. Un 60\% de bibliotecas están suscritas a publicaciones periódicas. El acceso a los documentos es libre en un 80\%. Para un correcto diseño hacia el CREA es fundamental 
que todos los recursos bibliográficos, documentales y multimedia estén recogidos, centralizados y gestionados en la biblioteca con el fin de ponerlos a disposición de la comunidad escolar, pero sólo un 50\% contesta afirmativamente. También la edición es un rasgo definitorio del CREA, pero sólo un 50\% de las bibliotecas elabora algún tipo de material informativo o pedagógico. Un 60\% tiene alguna herramienta de comunicación virtual.

Respecto a la resolución de las necesidades informativas, el comportamiento de profesores y alumnos es significativamente coincidente. Los profesores consideran sus necesidades resueltas siempre en un 25,6\%, y la mayoría de las veces un 56,4\%; los alumnos, por su parte, ven sus necesidades resueltas siempre entre un $18,1 \%$ y un $29,4 \%$ de las ocasiones, la mayoría de las veces entre un $53,1 \%$ y un $67,3 \%$, según el nivel educativo.

\section{Modelo ideal de biblioteca}

Se trata de un aspecto por el que sentíamos un gran interés, pero que por su propia naturaleza"modélica", no podía trasladarse fácilmente a un modelo evaluativo cuantitativo. Era necesario establecer un procedimiento para el tratamiento de la información cualitativa: primero se ha segmentado la muestra estadística en grupos-meta (alumnos, con sus niveles, profesores, directores, responsables), luego se han agrupado las respuestas por homogeneidad (asignando a cada una la frecuencia establecida según el nº de coincidencias, nunca menor a 5), y finalmente se han seleccionado las palabras-clave representativas de una biblioteca escolar modélica, que es lo que ahora presentamos.

a) Los alumnos: consideradas las palabras-clave que representan semánticamente la opinión de este grupo-meta se obtiene, como principales valores modélicos, en orden de relevancia: grande y amplia, con un alto número de ordenadores, tranquilidad y silencio, buena conexión a Internet, variedad de materiales o recursos, horario amplio.

b) Los profesores: la "idealización" de la biblioteca quedaría reflejada en su percepción como un centro dinámico e interdisciplinar donde coincidieran los espacios de lectura, estudio, trabajo en grupo e Internet, donde existiese una gran cantidad y diversidad de recursos disponibles para toda la comunidad educativa, un centro que apoyara la formación en la búsqueda y recuperación de la información y que estuviese gestionado por personal especializado.

c) Los responsables: las respuestas fueron mucho más lapidarias, concisas y unánimes y son realmente propuestas: un mayor apoyo al responsable; ser un centro de recursos organizado que utiliza cualquier tipo de soporte, apoya al aprendizaje activo y permite la igualdad educativa; ser centro de recursos con un fondo útil y actualizado al servicio de la comunidad escolar; ser biblioteca de consulta, lectura, trabajo, acceso libre a la red, en contacto virtual con otras bibliotecas.

d) Los directores: al igual que en el caso de los responsables, proponen respuestas más significativas: más amplia, con inclusión de la DVDteca; un centro de recursos documentales y multimedia; un lugar de encuentro, comunicación e intercambio de experiencias educativas; centro de recursos y servicios, activo y de información para la comunidad educativa; una biblioteca abierta la mayor parte del día; espacio donde se prolonga el espacio de enseñar y aprender; biblioteca integrada en la gran parte de las actividades del centro.

\section{Resultados y Discusión}

\section{Análisis de diagnóstico}

El estudio de campo demuestra la importancia decisiva que tiene el compromiso de los equipos directivos de los centros con las bibliotecas, por cuanto los directores se encargan de adjudicar un presupuesto a la biblioteca y poner a un profesor al frente de la misma. Es relevante que un alto porcentaje del profesorado, de los directores y de los responsables considere a la biblioteca un espacio de documentación, información y formación, así como que la totalidad de directores y responsables considera que apoyar el proceso de enseñanza-aprendizaje y lograr los objetivos de la compensación educativa forman parte de los objetivos de la biblioteca.

Por otro lado, existe una realidad insoslayable: en la mayoría de los centros se desconoce qué es en realidad un CREA y cuál es su alcance, observando además que 
no lo perciben del mismo modo los directores que los responsables, estando la percepción de estos últimos más ajustada a la realidad. De hecho, los centros que sí han iniciado la transformación al CREA son los que han tomado medidas como fomentar la creación de un equipo de biblioteca estable, coordinado por un responsable con formación específica, e integrar el proyecto de biblioteca en los proyectos educativos del centro.

Como en tantos aspectos de las Unidades de Información, el factor humano es determinante como base firme para iniciar una transformación a CREA, aunque sólo un 50\% de los directores y un 60\% de los responsables han aportado diversas opiniones a la viable transformación a CREA. Los responsables muestran bastante escepticismo por la lógica resistencia a que dicha transformación tenga que depender únicamente de su voluntarismo. El hecho de que un tercio del profesorado no considere que el CREA vaya a facilitar en los alumnos la adquisición de las competencias básicas tiene un valor a considerar en una posible transformación. Se hace necesario el compromiso de la Administración para crear la figura del bibliotecario escolar.

Otro escenario que debe tomarse en cuenta es el desarrollo de redes educativas o redes de bibliotecas escolares como el medio idóneo para un desarrollo sólido del CREA, garantizando un impacto saludable en la calidad de la Educación. Se detecta, en cambio, que apenas existe colaboración con otras bibliotecas, ya sean de otros centros del entorno o bibliotecas municipales. Esto redunda, en ocasiones, en una notoria falta de infraestructuras y recursos que los centros tienden a resolver presentando proyectos de mejora en concursos de carácter autonómico o nacional y solicitando ayudas a la Administración o a otros organismos. Este sistema ofrece resultados perversos: se dota a los centros de partidas específicas destinadas a las adquisiciones de material o equipamiento tecnológico para la biblioteca, pero sin criterios específicos de calidad y sin continuidad en el tiempo.

Los resultados de este estudio de campo muestran que, en la situación actual, las bibliotecas escolares en España, sin duda habiendo avanzado mucho y bien, están lejos de las Pautas que establece la International
Federation of Librarians Federation (IFLA). Este desfase exige un esfuerzo para adecuarse a las recomendaciones de la Unesco, para la que la biblioteca escolar es parte integrante del proceso educativo y asume unas funciones que permiten desarrollar los conocimientos básicos, los rudimentos en materia de información, la enseñanza, el aprendizaje y la cultura (International Federation of Library Associations, 2000).

Para cumplir las funciones que la IFLA establece en sus Pautas, la biblioteca escolar debe formular políticas y crear servicios, seleccionar y adquirir materiales, facilitar el acceso material e intelectual a las fuentes de información adecuadas, proporcionar recursos didácticos y emplear a personal capacitado. Son funciones que se hacen explícitas en el modelo CREA. Es un modelo, además, que se ha de orientar a servir de apoyo a una nueva concepción educativa centrada en el aprendizaje, articulando sus servicios en torno al currículo, lo que implica: a) promocionar la lectura, en todas sus variantes; b) proporcionar a la comunidad educativa las competencias necesarias para desenvolverse en la Sociedad del Conocimiento, apoyando e impulsando su Alfabetización en Información; c) representar un espacio plenamente adaptado a los entornos tecnológicos; d) organizar y representar el conocimiento expresado y difundido mediante un nuevo tipo de documento educativo, los objetos de aprendizaje; e) ser un servicio global e integrado de información, formación y orientación.

\section{Propuesta de un modelo de transformación a CREA}

El proyecto de investigación, como resultado, se proponía definir indicadores que permitan evaluar el modelo de transformación al CREA, proporcionando un método para la implantación del modelo, realizar su seguimiento, establecer pautas para las acciones a llevar a cabo y determinar su impacto. Cada indicador lleva implícita una toma de decisiones que será llevada a cabo por los responsables correspondientes. Los indicadores están estructurados en las siguientes categorías: I. Indicadores del proceso de transformación de biblioteca escolar a CREA; II. Indicadores de los niveles de percepción del CREA; III. Indicadores de la proyección del CREA en el currículum; IV. Indicadores de acceso a los recursos y a la información. 
1) Indicadores de proceso de transformación a CREA: son sus elementos de diseño del único indicador contemplado:

Definición: porcentaje de tareas CREA realizadas por la biblioteca escolar, según el modelo de transformación, de acuerdo a una escala gradual: valor $0 \%$ "No ha iniciado la transformación" y el valor 100\%, "CREA".

Objetivo: conocer en qué nivel de transformación a CREA se encuentra la biblioteca escolar con respecto a: Modelo de biblioteca; Recursos Humanos; Acceso a los recursos y a la información; Uso CREA de la biblioteca escolar.

Método: diseñar una lista con las diversas tareas estableciendo prioridades, comprobando cuántas tareas CREA se han cumplido y hallar el porcentaje.

II) Indicadores de niveles de percepción del CREA: los elementos de diseño se refieren dos indicadores contemplados:

- Desarrollo de actividades:

Definición: porcentaje de actividades y acciones CREA se llevan a cabo.

Objetivo: determinar la viabilidad que tiene el CREA de desarrollar actividades propias de sus funciones: gestión de los recursos didácticos y documentales; apoyo a la investigación y a la innovación docentes; formación y educación en Alfabetización en Información (ALFIN); promoción de la lectura en todas sus variantes; e-learning.

Método: el porcentaje se obtendrá calculando el grado de éxito de las tareas de cada actividad. Se presentarán las facilidades y obstáculos surgidos.

- Intervención del CREA en proyectos y programas del Centro:

Definición: porcentaje de programas y proyectos con el CREA.

Objetivo: conocer la presencia del CREA en los programas y proyectos del centro como parte integrante del currículum académico.

Método: el porcentaje se obtendrá por la proporción entre el total de proyectos, actuaciones y alumnos del centro y la intervención del CREA en los mismos.
III) Indicadores de proyección del CREA en el currículo académico: los elementos de diseño se refieren a tres indicadores propuestos:

- Complementariedad:

Definición: porcentaje de actividades didácticas con soporte del CREA.

Objetivo: determinar cuáles actividades de las programaciones didácticas de los Departamentos Didácticos necesitan ser sustentadas por el CREA, para detectar los Departamentos que consideran al CREA como elemento facilitador del proceso educativo. Las actividades hacen referencia a: proyectos de investigación aula - CREA; elaboración de Unidades Didácticas; asesoramiento al profesorado; préstamo de materiales al aula.

Método: el porcentaje será por Departamento y se obtendrá dividiendo el número de actividades con el apoyo del CREA entre el total de actividades.

- Formación del profesorado:

Definición: porcentaje de profesorado formado en el uso didáctico del CREA.

Objetivo: medir el impacto de la formación del profesorado en el uso del CREA relacionada con: ALFIN; elaboración de materiales didácticos; interacción aula-CREA; utilización de materiales educativos digitales.

Método: el porcentaje calculado respecto al número total de profesores.

- Uso de herramientas colaborativas:

Definición: porcentaje de profesorado que para su práctica docente hace uso de herramientas colaborativas, con auxilio del CREA.

Objetivo: determinar cuántos profesores ofrecen a sus alumnos herramientas de creación de materiales de aprendizaje (wikis, edición de página Web, mapas conceptuales, blog etc.) y herramientas para el trabajo en colaboración.

Método: porcentaje calculado sobre el número total de profesores del centro.

IV) Indicadores de acceso a los recursos de información: los elementos de diseño de indicadores se aplican a seis indicadores:

- Uso de servicios CREA: 
Definición: porcentaje de uso de los servicios prestados por el CREA a la comunidad educativa.

Objetivo: medir el uso de servicios de información, orientación y formación que el CREA ofrece a la comunidad educativa para determinar sus perspectivas de impulso y eficacia: Open Public Acess Catalog (OPAC), depósitos de e-revistas y e-book, acceso a diversas bases de datos, préstamos, consulta en sala, formación-educación usuarios, difusión de información, orientación bibliográfica etc.

Método: el porcentaje se obtendrá por la proporción que existe entre el uso de cada servicio prestado y el total de servicios que presta la biblioteca CREA.

- Infraestructura tecnológica:

Definición: porcentaje de ordenadores reales y potenciales con conexión Internet en relación al número de alumnos y profesores.

Objetivo: medir el uso de la infraestructura tecnológica por el número real y potencial de ordenadores disponibles y conocer sus posibilidades tecnológicas.

Método: el porcentaje se calculará dividiendo el número total de ordenadores entre el total de alumnos y profesores del centro, así como el incremento.

\section{- Utilización del CREA:}

Definición: porcentaje de usuarios que han utilizado cualquiera de los servicios disponibles en el CREA.

Objetivo: determinar la competencia del CREA en la prestación de servicios según el acceso de los usuarios a los mismos.

Método: diseño de un cuestionario sobre el uso del CREA o sus servicios.

- Factibilidad de los recursos:

Definición: porcentaje de recursos informáticos, electrónicos, digitales e impresos demandados por la comunidad educativa.

Objetivo: determinar qué recursos solicitados por la comunidad educativa puede ofrecer el CREA, acorde a las necesidades de información de la comunidad educativa.

Método: estudio de usuarios para detectar las necesidades de información.

\section{- Acceso a la Web del CREA:}

Definición: porcentaje de visitas a la página Web del CREA.

Objetivo: medir el número de visitas que recibe la página Web del CREA para determinar la idoneidad de sus contenidos y el grado de satisfacción del usuario.

Método: incorporación de un contador en la página Web y cuestionarios de satisfacción online.

- Colaboración:

Definición: porcentaje de actividades realizadas por el CREA en colaboración con otras Unidades de Información.

Objetivo: determinar las Unidades de Información con las que colabora el CREA (Bibliotecas Públicas, Municipales, Universitarias, Privadas, de otros centros educativos etc.) y establecer el número de actividades realizadas, el carácter de las mismas, el índice de participación, la duración.

Método: el porcentaje se calculará dividiendo el total de actividades realizadas por el CREA entre el total de actividades en colaboración.

\section{Aplicación práctica en el marco del ámbito escolar}

El desarrollo del modelo de transformación reclama unos elementos aplicativos:

Formación ALFIN para el profesorado: los resultados muestran que parte del profesorado carece de formación en ALFIN y en el uso didáctico de la biblioteca-CREA, lo cual es un grave obstáculo ya que, en opinión de Sánchez Tarragó (2005), el profesor, protagonista activo en la formación de competencias informacionales, debe tener formación específica en los usos de las tecnologías educativas y en trabajo multidisciplinario e innovación, así como integrar estos procesos en el trabajo en el aula.

El profesorado necesita un cambio en su metodología, más enfocada al trabajo en equipo y con un carácter más dinámico e innovador. Moreno Rodriguez (2007) presenta bloques de contenido que definen los aspectos que la formación del profesorado debe contemplar en el ámbito de la biblioteca-CREA: a) el conocimiento de los recursos, servicios y posibilidades 
que ofrece para complementar y enriquecer la labor docente; b) elaboración de actividades en función de los recursos disponibles en el CREA y del alumnado; c) conocimiento, manejo y uso de programas orientados a enseñar, a investigar y a alfabetizar informacionalmente al alumnado; d) conocimiento del uso y tratamiento de la información, las técnicas de trabajo intelectual y la instrucción documental, las estrategias para expresarse por escrito, mediante el análisis; e) utilización de estrategias de investigación, creación de materiales, innovación educativa, trabajo en equipo para la mejora de la capacidad de la organización escolar en la utilización de los recursos bibliotecarios.

No podemos dejar de aludir a las posibilidades que ofrece el e-learning como una estrategia formativa que puede resolver muchos de los problemas con los que se encuentra el profesorado a la hora de mostrar interés por la realización de un curso específico de formación. Es una modalidad de aprendizaje no presencial, interactiva y generalmente económica, que facilita la comunicación profesor-alumno a través de herramientas asincrónicas como el e-mail y sincrónicas como el chat, flexible en su realización.

Actividades para desarrollar la alfabetización en información y la digital: los alumnos, sin duda, poseen notorias destrezas en el manejo de la tecnología; sin embargo, los mismos carecen de habilidades informacionales porque no saben usarla adecuadamente. Los alumnos necesitan aprender a manejar los contenidos, a organizar e interpretar la información con el fin de convertirla en conocimiento (Durban Roca, 2007). Al finalizar la Educación Secundaria, el alumno debe dominar las habilidades de búsqueda documental: saber dónde y cómo hallar la información necesaria, así como estar capacitado para resolver los problemas que se le planteen o tomar decisiones (Andreu, 2006).

A este respecto, los proyectos documentales integrados, de carácter multidisciplinar, son investigaciones que permiten al alumno familiarizarse con los mecanismos de búsqueda de información y trabajo intelectual empleando los recursos del CREA y donde el profesor hará unas veces de guía y otras de fuente (Piquín, 2005). El establecimiento, programación y desarrollo de actividades pasa por dos procedimientos que tienen en común el uso de la información: trabajos de investigación planteados por los Departamentos Didácticos y que requieren utilizar el CREA para realizarlos y actividades que directamente organiza el CREA. Ambos contemplan la interacción aula-CREA y la coordinación entre profesor y bibliotecario.

Recursos y materiales didácticos digitales: I calidad y utilidad de los materiales y recursos en red pueden plantear ciertos problemas a la hora de su utilización, porque ¿cómo se puede garantizar su rigor científico? Más aún, a pesar de tener calidad suficiente ¿es adecuado para su utilización en el aula? (Martín-Laborda, 2005). Esto suscita la conveniencia o no de crear un organismo que evalúe los nuevos materiales educativos, factor que encuentra una diversidad de opiniones al respecto y que nos pone en la pista de unos indicadores de credibilidad entre los que algunos investigadores apuntan: identidad de los autores, la usabilidad, la claridad del lenguaje utilizado, la adecuación del diseño, la frecuencia de actualizaciones, los enlaces y las aplicaciones y herramientas que utiliza.

A modo de propuesta indicativa de recursos digitales educativos apropiados para un CREA en España, apuntamos: Ia Biblioteca Escolar Digital (http:// www.bibliotecaescolardigital.es/), herramienta que permite compartir, utilizar, crear, difundir, evaluar y recomendar objetos digitales educativos de calidad con garantía de acceso universal; Thinkquest (http:// www.thinkquest.org/es/), plataforma de aprendizaje en la que maestros y estudiantes crean proyectos de aprendizaje; Jclic (http://clic.xtec.cat/es), aplicación desarrollada en la plataforma Java para el diseño de contenidos de aprendizaje; Neobook (http://neobook. programas-gratis.net), programa que sirve para la creación de contenidos educativos; Agrega (http://agrega. educacion.es/), red abierta de contenidos educativos digitales; Instituto de Tecnología Educativa (ITE) (http:// www.ite.educacion.es/), unidad del Ministerio de Educación responsable de la integración de las TIC en las etapas educativas no universitarias; eTwinning (http:// www.etwinning.es/), portal que pretende dar a conocer los proyectos y experiencias escolares y hacer visibles las buenas prácticas educativas en Europa. 


\section{Referencias}

ANDREU, L.B. La biblioteca del IES como centro de recursos para el aprendizaje y la enseñanza (CRAE). Leer.es, p.39-51. 2006. Disponible en: <http://www.ite.educacion.es/w3/ recursos2/ bibliotecas/bibliotecas_2/buenaspracticas2006/ la_biblioteca_del_ies.pdf>. Acceso en: 19 abr. 2012.

AREA, M. et al. De las bibliotecas universitarias a los centros de recursos para el aprendizajey la investigación: resumen informe. Madrid: REBIUN-CRUE, 2005

DURBAN ROCA, G. Articulación de programas de educación en información desde la biblioteca escolar: aprender a investigar e informarse. In: INSTITUTO SUPERIOR DE FORMACIÓN DEL PROFESORADO (Org). La articulación de los recursos en el funcionamiento de la biblioteca escolar. Madrid: Instituto Superior de Formación del Profesorado, 2007. p.133-158.

ESPAÑA. Orden ECI/2220, de 12 de julio de 2007. Por la que se establece el currículo y se regula la ordenación de la educación secundaria obligatoria. Boletín Oficial del Estado, 21 jul. 2007. n.174, p.31680.

INTERNATIONAL FEDERATION OF LIBRARY ASSOCIATIONS. Manifiesto UNESCO/IFLA sobre la biblioteca escolar. 2000. Disponible en: <http://www.unesco.org/webworld/libraries/ manifestos/school_manifesto_es.html>. Acceso en: 19 abr. 2012.

MADRID. Subdirección General de Bibliotecas. Plan de bibliotecas escolares en red: desarrollo. Madrid: Subdirección General de Bibliotecas, 2007. Disponible en: <http:// www.madrid.org/cs/Satellite?blobcol=urldata \&blobheader=application\%2Fpdf\&blobheadername1=ContentDisposition\&blobheadervalue 1=filename\%3DPLAN+noviembre+
2007.pdf\&blobkey=id\&blobtable=MungoBlobs\& blobwhere $=1220528988962 \&$ ssbinary $=$ true $>$. Acceso en: 19 abr. 2012

MARTÍN-LABORDA, R. Las nuevas tecnologías en educación. Madrid: Fundación AUNA, 2005. Disponible en: <http:// www.fundacionorange.es/documentos/analisis/cuadernos/ cuadernos_05_rocio.pdf>. Acceso en: 19 abr. 2012.

MARZAL, M.A.; CUEVAS, A. Biblioteca escolar para la sociedad del conocimiento en España. Ciência da Informação, v.36, n.1, p.54-68, 2007. Disponible en: <http://revista.ibict.br/ciinf/ index.php/ciinf/article/view/909/707>. Acceso em: 19 abr. 2012.

MORENO RODRÍGUEZ, E.E. Recursos humanos, materiales y elementos clave de los planes de trabajo de la biblioteca escolar. In: MORENO RODRÍGUEZ, E.E. La articulación de los recursos en el funcionamiento de la biblioteca escolar. Madrid: Instituto Superior de Formación del Profesorado, 2007. p.57-88.

PIQUÍN, R. Proyectos documentales integrados: una experiencia tecnológica entre bibliotecas y escuelas. In: PIQUÍN, R. ¿Nuevas lecturas? ¿Nuevas formas de leer?: lectura y escritura multimedia en las bibliotecas públicas y escolares. 2005. Disponible en: <http://sol-e.com/plec/archivos/ Docs_Bibliografias/rpiquin.pdf>. Acceso en: 19 abr. 2012.

SÁNCHEZ TARRAGÓ, N. El profesional de la información en los contextos educativos de la sociedad del aprendizaje: espacios y competencias. Acimed, v.13, n.2, 2005. Disponible en:<http://bvs.sld.cu/revistas/aci/vol13_2_05/aci02_05.htm>. Acceso en: 19 abr. 2012. 